\title{
Application of $\zeta$-factor Microanalysis to Measure Phase Compositions in Ultrahard Ceramics and Complex Concentrated Alloys
}

Christopher Marvel ${ }^{1}$, Joshua Smeltzer ${ }^{2}$, Kristopher Behler ${ }^{3}$, B. Chad Hornbuckle ${ }^{3}$, Jerry LaSalvia ${ }^{3}$, Kristopher Darling $^{3}$, Masashi Watanabe ${ }^{1}$ and Martin Harmer ${ }^{2}$

${ }^{1}$ Lehigh University, Bethlehem, Pennsylvania, United States, ${ }^{2}$ Lehigh University, United States, ${ }^{3}$ Army Research Laboratory, United States

$\zeta$-factor microanalysis is a (scanning) transmission electron microscopy ((S)TEM) X-ray energy dispersive spectroscopy (XEDS) quantification method that corrects for specimen thickness effects [1]. It has been recently extended to compositionally characterize ultrahard ceramics [2] and complex concentrated alloys [3]. This talk will review $\zeta$-factor microanalysis, then describe the difficulties and subsequent strategies to characterize ultrahard ceramics and complex concentrated alloys.

Ultrahard ceramics (e.g. boron carbide) are used for high-strength applications. Methods to improve their performance focus on engineering their microstructure across multiple length-scales, particularly modifying bulk stoichiometry, incorporating nanoscale phases, developing stacking faults, and engineering grain and phase boundaries [4]. The approach of tailoring nanoscale phases and interfaces requires high-resolution techniques (e.g. (S)TEM). However, there are significant challenges of analyzing ultrahard ceramics using (S)TEM XEDS, namely poor detectability and high absorption loss of soft X-ray lines, native surface oxide layers, hydrocarbon contamination, etc. In combination, these analytical difficulties often result in inconclusive (S)TEM XEDS results. $\zeta$-factor microanalysis has the capability to overcome these experimental difficulties because of its inherent absorption correction capability.

The first task was to determine the $\zeta$-factors associated with the EDS detector in our JEOL ARM-200CF instrument. K-family $\zeta$-factors were initially determined or estimated using a NIST SRM-2063a thin film. Wedged $\mathrm{SiB}_{6}, \mathrm{SiC}$, and $\mathrm{GaN}$ thin specimens were also used to directly determine $\mathrm{B}, \mathrm{C}$, and $\mathrm{N} \mathrm{K}$-line $\zeta$-factors [2]. Figure 1a shows the $\zeta$-factors generated during this work. Most notably, direct measurement of $\mathrm{B}, \mathrm{C}$, and $\mathrm{N} \mathrm{K}$-line $\zeta$-factors revealed that indirect estimation methods underestimate their true value. Next, the $\mathrm{B}$ and $\mathrm{C}$ $\mathrm{K}$-line $\zeta$-factors were validated by analyzing three standard boron carbide specimens having different $\mathrm{B} / \mathrm{C}$ ratios, namely $\mathrm{B}_{10.0} \mathrm{C}, \mathrm{B}_{7.4} \mathrm{C}$, and $\mathrm{B}_{4.1} \mathrm{C}$, where the standard boron carbide compositions were independently determined via titration and combustion gas measurements [5]. STEM XEDS spectrum-imaging datasets were acquired from each bulk sample to efficiently collect thousands of individual spectra from different specimen thicknesses. Acquisition of spectra from different specimen thicknesses was conducted to confirm that $\zeta$-factor microanalysis can accurately, and precisely, determine boron carbide stoichiometry despite specimen thickness effects. Figure $1 \mathrm{~b}$ shows histograms of $\mathrm{B} / \mathrm{C}$ concentration ratios obtained from each boron carbide specimen. Overall, the $\zeta$-factor results agreed well with the known boron carbide stoichiometries, thus proving $\zeta$-factor microanalysis as a viable method of determining stoichiometries of ultrahard ceramics [2].

Complex concentrated alloys (CCAs) oftentimes exhibit unpredictable microstructures that are highly dependent on synthesis/processing methods. In some cases, CCAs exhibit multi-phase microstructures that consist of intentional metallic phases as well as unintentional impurity ceramic phases. Therefore, there is a need to confidently identify microstructural phases in CCAs in order to elucidate mechanical properties. Similar to ultrahard ceramics, difficulties associated with analyzing ceramic phases in CCAs via STEM XEDS are poor detectability and high X-ray absorption of soft $\mathrm{C}, \mathrm{N}$, and $\mathrm{O} \mathrm{K}$-line X-rays. In addition, poor detectability of very hard metallic X-ray lines (e.g. Nb K $\alpha$ and $\mathrm{Mo} \mathrm{K} \alpha$ ) and overlapping X-ray peaks also add to the difficulty. Considering the challenges of XEDS characterization of CCAs, a refractory NbMoTaW alloy 
that contains multiple impurity nitride phases was chosen as a model alloy to showcase the capabilities of $\zeta$ factor microanalysis.

The NbMoTaW alloy was synthesized by cryogenic ball milling and combustion gas methods determined that the alloy contained 25 at.\% N. Impurity nitrogen was introduced by milling with $\mathrm{LN}_{2}$. The alloy was then annealed at $1200{ }^{\circ} \mathrm{C}$ for 100 hours to induce grain growth. An XEDS spectrum-image was acquired and the results are shown in Figure 2. Maps of specimen thickness and all atomic concentrations of the major intentional and unintentional alloying elements were extracted. The specimen thickness map shows that the sample was roughly $100 \mathrm{~nm}$ thick towards the top left of the image but nearly 10-20 nm towards the bottom. Despite the great range of specimen thicknesses, and thereby differences in varying absorption losses for each element, the atomic concentration maps do not show a dependence on specimen thickness. The $\mathrm{N}$ and $\mathrm{Nb}$ atomic fraction maps, shown in Figure $2 \mathrm{c}$ and 2e, respectively, clearly show that two nitride phases precipitated: one enriched with $\mathrm{N}-\mathrm{Nb}-\mathrm{Ta}$ (marked by white circles) and another enriched with $\mathrm{N}-\mathrm{Fe}-\mathrm{Nb}-\mathrm{Ta}$ (marked by while triangles). The experimentally determined nitrogen concentrations in the two nitrides were $14.6 \pm 0.6$ and $31.3 \pm 0.9$ at. $\% \mathrm{~N}$, respectively. Atomic-resolution STEM imaging was also conducted to aid in the nitride phase identification. Images were taken from several low index zone axes and it was determined that nitride phases were cubic and hexagonal. Upon considering the nitrogen concentrations and crystal structures, the nitride phases were determined to be a $\mathrm{M}_{6} \mathrm{~N} \eta$-nitride and a $\mathrm{M}_{2} \mathrm{~N}$ heminitride. Their theoretical nitrogen concentrations of 14.3 and 33.3 at.\% $\mathrm{N}$, respectively, were in close agreement with the $\zeta$ factor measurements. Overall, $\zeta$-factor microanalysis enabled full phase identification which would otherwise have been difficult due to severe X-ray absorption [3].
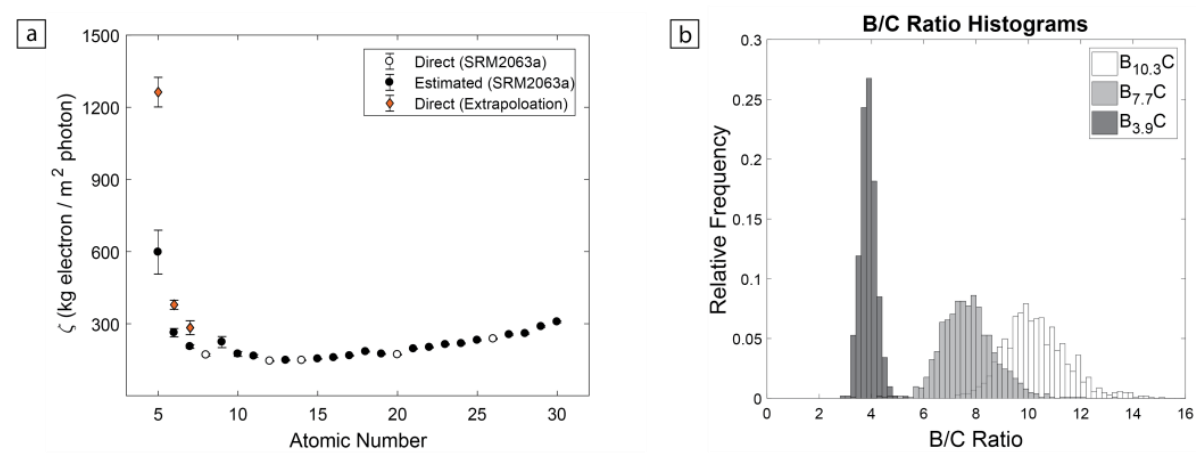

Figure 1. (a) Experimentally determined $\zeta$-factors and (b) determined boron carbide stoichiometries of three bulk samples.
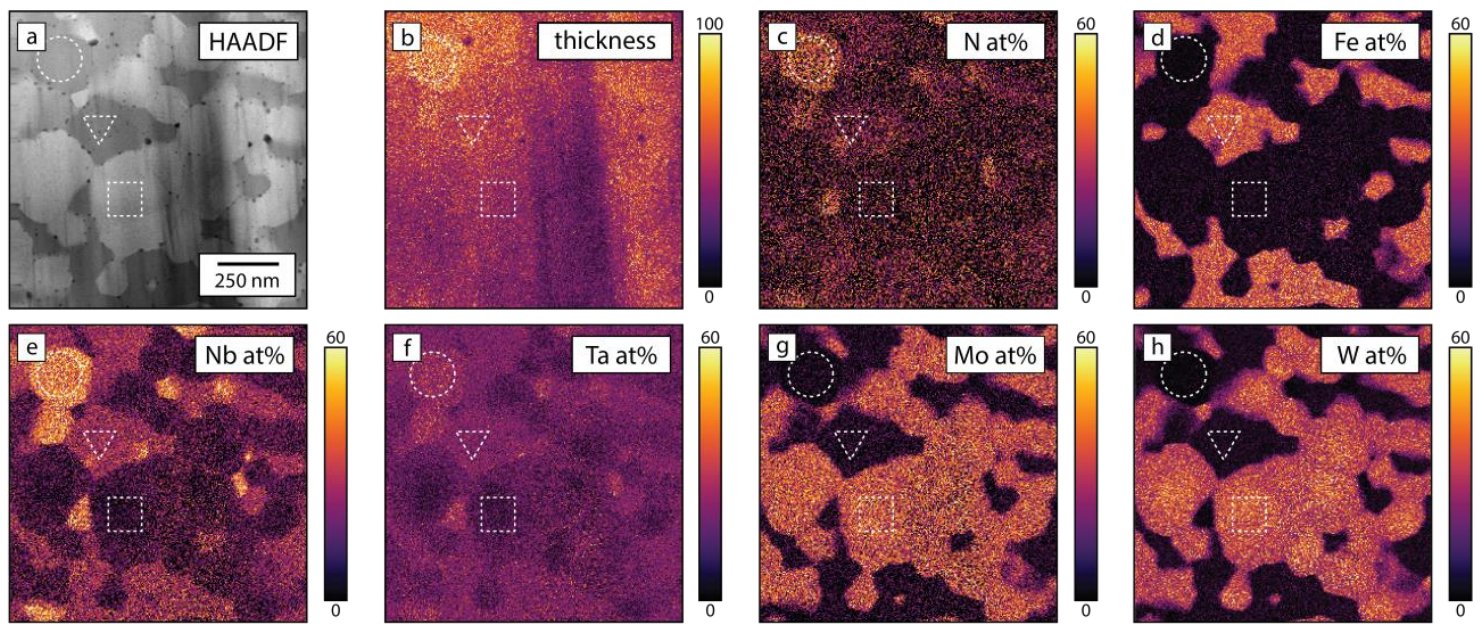
Figure 2. STEM-XEDS spectral image of a mechanically alloyed NbMoTaW CCA after annealing at $1200{ }^{\circ} \mathrm{C}$ for 1000 hours: (a) HAADF micrograph, (b) thickness (nm), (c) N at.\%, (d) Fe at.\%, (e) Nb at.\%, (f) Mo at.\%, (g) Ta at.\%, and (h) W at.\%. The microstructure contained a W(Mo) BCC solid solution (square), Fe-Ta-Nbrich M6N $\eta$-nitride (triangle), and a Nb-Ta-rich $\mathrm{M} 2 \mathrm{~N}$ heminitride (circle). Mo and $\mathrm{W}$ were correlated just as $\mathrm{Ta}$ and $\mathrm{Nb}$ were correlated.

\section{References}

[1] M. Watanabe and D. B. Williams. "The quantitative analysis of thin specimens: a review of progress from the Cliff-Lorimer to the new $\zeta$-factor methods." Journal of microscopy 221, no. 2 (2006): 89-109.

[2] C.J. Marvel, K. D. Behler, J. C. LaSalvia, V. Domnich, R. A. Haber, M. Watanabe, and M. P. Harmer. "Extending $\zeta$-factor microanalysis to boron-rich ceramics: Quantification of bulk stoichiometry and grain boundary composition." Ultramicroscopy 202 (2019): 163-172.

[3] J.A. Smeltzer, C.J. Marvel, B.C. Hornbuckle, A.J. Roberts, J.M. Marsico, A.K. Giri, K.A. Darling, J.M. Rickman, H.M. Chan, and M.P. Harmer. "Achieving ultrahard refractory multi-principal element alloys via mechanical alloying." Materials Science and Engineering: A 763 (2019): 138140.

[4] K.D. Behler, C.J. Marvel, J.C. LaSalvia, S.D. Walck, M.P. Harmer. "Observations of grain boundary chemistry variations in a boron carbide processed with oxide additives." Scripta 142 Materialia (2018): 10610.

[5] K.A. Kuwelkar. "Chemical and structural characterization of boron carbide powders and ceramics." Dissertation, Rutgers University (2016). 